\title{
Symplectic Methods for Separable Hamiltonian Systems
}

\author{
Mark Sofroniou $^{1}$ and Giulia Spaletta ${ }^{2}$ \\ 1 Wolfram Research, Champaign, Illinois, USA. marks@wolfram.com \\ 2 Mathematics Department, Bologna University, Italy. giulia@cs.unibo.it
}

\begin{abstract}
This paper focuses on the solution of separable Hamiltonian systems using explicit symplectic integration methods. Strategies for reducing the effect of cumulative rounding errors are outlined and advantages over a standard formulation are demonstrated. Procedures for automatically choosing appropriate methods are also described.
\end{abstract}

Keywords. Geometric numerical integration; separable Hamiltonian differential equations; symplectic methods; computer algebra systems.

AMS. 65L05; 68Q40.

\section{Introduction}

The phase space of a Hamiltonian system is a symplectic manifold on which there exists a natural symplectic structure in the canonically conjugate coordinates. The time evolution of the system is such that the Poincaré integral invariants associated with the symplectic structure are preserved. A symplectic integrator is advantageous because it computes exactly, assuming infinite precision arithmetic, the evolution of a nearby Hamiltonian, whose phase space structure is close to that of the original Hamiltonian system [11].

Symplectic integration methods for general Hamiltonians are implicit, but for separable Hamiltonians explicit methods exist and are much more efficient [23]. The aim of this work is to describe a uniform framework that provides a variety of numerical solvers for separable Hamiltonian differential equations in a modular, extensible way. Furthermore, the effect of rounding errors has not received a great deal of attention, so the framework is used to explore this issue in more detail.

This paper is organized as follows. In Section 2 separable Hamiltonian systems are defined together with a standard algorithm for implementing an efficient class of symplectic integrators. Practical algorithms for reducing the effect of cumulative rounding errors are presented in Section 3. Section 4 contains a description of the methods that have been implemented, along with algorithms for automatically selecting between different orders and a procedure for adaptively refining coefficients for high precision computation. Section 5 contains some numerical experiments that summarize the behavior of the various algorithms presented. Section 6 concludes with some potential enhancements and suggestions for future work. 


\section{Definitions}

Let $\Omega$ be a nonempty, open, connected subset in the oriented Euclidean space $\mathbb{R}^{2 d}$ of the points $(\mathbf{p}, \mathbf{q})=\left(p_{1}, \ldots, p_{d}, q_{1}, \ldots, q_{d}\right)$. Denote by $I$ an open interval of the real line. If $H=H(\mathbf{p}, \mathbf{q}, t)$ is a sufficiently smooth real function defined on the product $\Omega \times I$, then the Hamiltonian system of differential equations with Hamiltonian $\mathrm{H}$ is:

$$
\frac{d p_{i}}{d t}=-\frac{\partial H}{\partial q_{i}}, \quad \frac{d q_{i}}{d t}=\frac{\partial H}{\partial p_{i}}, \quad i=1, \ldots, d .
$$

The integer dimension $d$ is referred to as the number of degrees of freedom and $\Omega$ as the phase space.

Many practical problems can be modeled by a separable Hamiltonian where: $H(\mathbf{p}, \mathbf{q}, t)=T(\mathbf{p})+V(\mathbf{q}, t)$. The Hamiltonian system can then be expressed in partitioned form by means of two functions $\mathbf{f}$ and $\mathbf{g}$ :

$$
\frac{d p_{i}}{d t}=\mathbf{f}\left(q_{i}, t\right)=-\frac{\partial V(\mathbf{q}, t)}{\partial q_{i}}, \quad \frac{d q_{i}}{d t}=\mathbf{g}\left(p_{i}\right)=\frac{\partial T(\mathbf{p})}{\partial p_{i}}, \quad i=1, \ldots, d .
$$

A Partitioned Runge Kutta method (PRK) can be used to numerically integrate (1). In most practical situations the cost of evaluating $\mathbf{f}$ dominates the cost of evaluating $\mathbf{g}$.

Symplecticity is a characterization of Hamiltonian systems in terms of their solutions and it is advantageous if a numerical integration scheme applied to (1) is also symplectic. A Symplectic Partitioned Runge Kutta (SPRK) method involves constraints on the coefficients of a PRK method which results in a reduction in the number of order conditions that need to be satisfied [20]. Symplecticity also gives rise to a particularly simple implementation [21]. Following [23], denote the coefficients of an $s$ stage SPRK method as $\left[b_{1}, b_{2}, \ldots, b_{s}\right]\left(B_{1}, B_{2}, \ldots, B_{s}\right)$. Algorithm 1 yields an explicit integration procedure starting from initial conditions $\mathbf{p}_{n}, \mathbf{q}_{n}$.

\section{Algorithm 1 (Standard formulation)}

$$
\begin{aligned}
& \mathbf{P}_{0}=\mathbf{p}_{n} \\
& \mathbf{Q}_{1}=\mathbf{q}_{n} \\
& \text { for } i=1, \ldots, s \\
& \qquad \mathbf{P}_{i}=\mathbf{P}_{i-1}+h_{n+1} b_{i} \mathbf{f}\left(\mathbf{Q}_{i}, t_{n}+C_{i} h_{n+1}\right) \\
& \quad \mathbf{Q}_{i+1}=\mathbf{Q}_{i}+h_{n+1} B_{i} \mathbf{g}\left(\mathbf{P}_{i}\right)
\end{aligned}
$$

The algorithm returns $\mathbf{p}_{n+1}=\mathbf{P}_{s}$ and $\mathbf{q}_{n+1}=\mathbf{Q}_{s+1}$. The time weights are given by:

$$
C_{j}=\sum_{i=1}^{j-1} B_{i}, \quad j=1, \ldots, s .
$$

Two $d$ dimensional vectors can be used to implement Algorithm 1 [21], although practically three vectors may be necessary if the function call is implemented as a subroutine and cannot safely overwrite the argument data. If $B_{s}=0$ then Algorithm 1 effectively reduces to an $s-1$ stage scheme since it has a First Same As Last (FSAL) property. 


\section{Rounding error accumulation}

Errors are asymptotically damped when numerically integrating dissipative systems. Hamiltonian systems, on the contrary, are conservative and the Hamiltonian is a constant, or invariant, of the motion. Consequently, an issue when numerically integrating such systems is that errors committed at each integration step can accumulate. Furthermore, solutions of Hamiltonian systems often require very long time integrations so that the cumulative roundoff error can become important. Finally, high order symplectic integration methods also involve many basic sub steps and the form of Algorithm 1 means that rounding errors are compounded during each integration step. For these reasons it is useful to look for alternatives. In certain cases, Lattice symplectic methods exist and can avoid step by step roundoff accumulation, but such an approach is not always possible [6].

A technique for reducing the effect of cumulative error growth in an additive process is compensated summation (see [14] for a summary). In IEEE double precision, compensated summation uses two variables to represent a sum and an error, which has the effect of doubling the working precision. As illustration consider $n$ steps of a numerical integration using Euler's method, with a fixed step size $h$, applied to an autonomous system. The updates $\mathbf{y}+h \mathbf{f}(\mathbf{y})$ are replaced by results of the following algorithm.

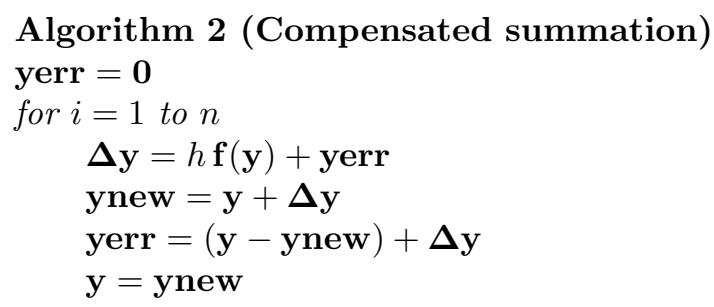

Traditionally compensated summation has been considered for dissipative systems when the step size is small [4], [14]. However the technique can be particularly useful for conservative systems, where errors are not damped asymptotically, even when the step size is relatively large [15]. For Hamiltonian systems, Algorithm 2 requires an extra two $d$ dimensional vectors over Algorithm 1 in order to store the rounding errors in updating $\mathbf{p}$ and $\mathbf{q}$. In a practical implementation in $\mathrm{C}$, the volatile declaration avoids computations being carried out in extended precision registers.

Compensated summation can be used directly at each of the internal stages in Algorithm 1. However, other possibilities exist. Our preference is to use Algorithm 3 below with compensated summation applied to the final results. This approach yields some small arithmetic savings and is more modular: it also applicable if the basic integrator is a symplectic implicit Runge-Kutta method.

As an example, consider evaluating $\sum_{k=1}^{n} x$ in IEEE double precision, with $x=0.1$ and $n=10^{6}$. Since 0.1 is not exactly representable in binary, a representation error is made at the outset. More importantly, the scale of the cumulative sum increases and this causes successive bits from each term added in 
Table 1. Default SPRK methods and a summary of their properties.

\begin{tabular}{|c|c|c|c|c|}
\hline Order & f Evals & Method & Symmetric & FSAL \\
\hline 1 & 1 & Symplectic Euler & No & No \\
\hline 2 & 1 & Symplectic pseudo Leapfrog & Yes & Yes \\
\hline 3 & 3 & McLachlan-Atela [16] & No & No \\
\hline 4 & 3 & Forest-Ruth [7], Yoshida [25], Candy-Rozmus [5] & Yes & Yes \\
\hline 6 & 7 & Yoshida A [25] & Yes & Yes \\
\hline 8 & 15 & Yoshida D [25] & Yes & Yes \\
\hline
\end{tabular}

to be neglected. Without compensated summation the result that we obtain is 100000.0000013329, while with compensated summation it is 100000 .

A reduction in compound numerical errors can be accomplished for SPRK methods by introducing the increments $\boldsymbol{\Delta} \mathbf{P}_{i}=\mathbf{P}_{i}-\mathbf{p}_{n}$ and $\boldsymbol{\Delta} \mathbf{Q}_{i}=\mathbf{Q}_{i}-\mathbf{q}_{n}$ and using the following modified algorithm.

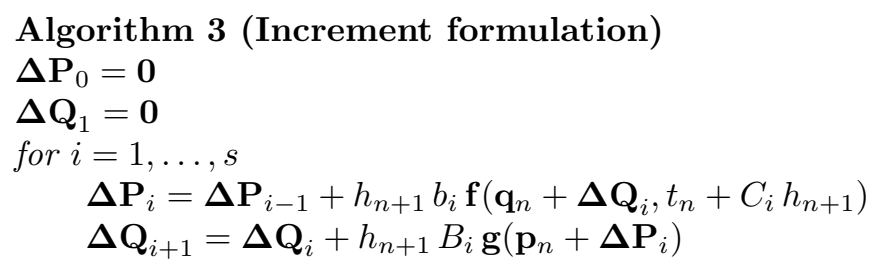

Algorithm 3 can be implemented using three extra $d$ dimensional storage vectors over Algorithm 1, as well as some additional elementary arithmetic operations. Two vectors are sufficient if the function can safely overwrite the argument data. An advantage over Algorithm 1 is that each of the quantities added in the main loop are now of magnitude $O(h)$. Furthermore instead of returning $\mathbf{p}_{n}+\boldsymbol{\Delta} \mathbf{P}_{s}$ and $\mathbf{q}_{n}+\mathbf{\Delta} \mathbf{Q}_{s+1}$, Algorithm 3 returns the increments of the new solutions and these can be added to the initial values using compensated summation. The additions in the main loop in Algorithm 3 could also be carried out employing compensated summation, but our experiments have shown that this adds a nonnegligible overhead for a relatively small gain in accuracy.

\section{Methods and order selection}

The default SPRK methods at each order in our current implementation are given in Table 1. The modularity also makes it possible for a user to select an alternative method by simply entering the coefficients and indicating the order. Only methods of order four or less in Table 1 possess a closed form solution. Higher order methods are given as machine precision coefficients. Since our numerical integration solver also works for arbitrary precision, we need a process for obtaining the coefficients to the same precision as that to be used in the solver. When the closed form of the coefficients is not available, the order equations 
for the symmetric composition coefficients can be refined in arbitrary precision using the Secant method, starting from the known machine precision solution.

In our framework, users can select the order of an SPRK method by specifying an option, but an automatic selection is also provided. To accomplish this, two cases need to be considered, leading to Algorithm 4 and Algorithm 5 below. The first ingredient for both algorithms is a procedure for estimating the starting step size $h_{k}$ to be taken for a method of order $k$ (see [8], [10]). The initial step size is chosen to satisfy user specified absolute and relative local tolerances. The next ingredient is a measure of work for a method.

Definition 1 (Work per unit step). Given a step size $h_{k}$ and a work estimate $W_{k}$ for one integration step with a method of order $k$, the work per unit step is given by $W_{k} / h_{k}$.

For an SPRK method of order $k$ the work per step is the number of function evaluations, effectively given by the number of stages $s(s-1$ if the FSAL device is used). The last ingredient is a specification of the methods that are available. Let $\Pi$ be the non empty, ordered set of method orders that are to be selected amongst. A comparison of work for the methods in Table 1 gives the choice $\Pi=\{2,4,6,8\}$. Denote $\Pi_{k}$ as the $k$-th element of $\Pi$ and $|\Pi|$ as the cardinality.

Of the two cases to be considered, probably the most common is when the step size can be freely chosen. The task is to compute a starting step and balance it with an estimate of the cost of the method. By bootstrapping from low order, Algorithm 4 finds the order that locally minimizes the work per unit step.

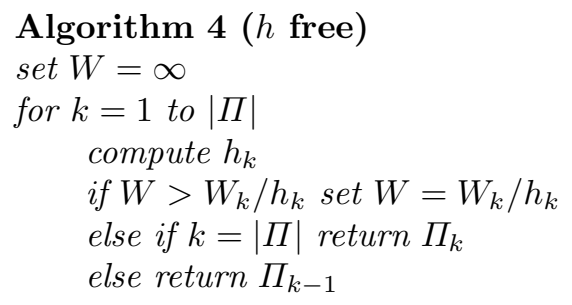

The second case to be considered is when the starting step size estimate $h$ is given. Algorithm 5 provides the order of the method that minimizes the computational cost while satisfying the given tolerances.

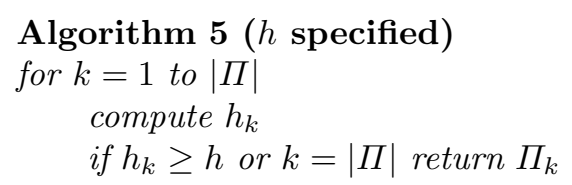

The computation of $h_{1}$ usually involves estimation of derivatives obtained from a low order integration (see [10, Section II.4]). The derivative estimates are the same for all $k>1$ and can therefore be computed only once and stored. Computing $h_{k}$, for $k>1$, then involves just a few basic arithmetic operations which are independent of the cost of function evaluation of the differential system.

Algorithms 4 and 5 are heuristic since the optimal step size and order may change through the integration, but symplectic integration commonly involves 


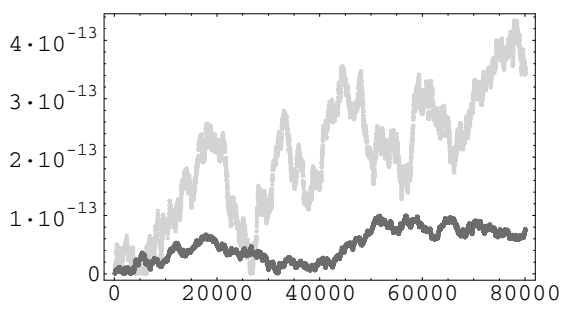

Fig. 1. Harmonic oscillator. Relative error growth vs time using Algorithm 1 (above) and Algorithm 3 (below).

fixed choices. In spite of this, both algorithms incorporate salient integration information, such as local error tolerances, system dimension and initial conditions, to avoid a poor default choice.

\section{Numerical examples}

The coefficients given in [25] are only accurate to 14 decimal digits, while those used in this section are accurate to full IEEE double precision. All computations are carried out using a developmental version of Mathematica.

\subsection{The Harmonic oscillator}

The Harmonic oscillator is a simple Hamiltonian problem that models a material point attached to a spring. For unitary mass and spring constant, the Hamiltonian is $H(p, q)=\left(p^{2}+q^{2}\right) / 2$, for which the differential system is:

$$
q^{\prime}(t)=p(t), \quad p^{\prime}(t)=-q(t), \quad q(0)=1, \quad p(0)=0 .
$$

The constant step size taken is $h=1 / 25$ and the integration is performed over the interval $[0,80000]$ with the 8 th order integrator in Table 1 . The error in the Hamiltonian is sampled every 200 integration steps. The exact solution evolves on the unit circle, but a dissipative numerical integrator produces a trajectory that spirals to the fixed point at the origin and exhibits a linear error growth in the Hamiltonian.

Figures 1, 2 and 3 show the successive improvement in the propagated error growth using Algorithm 1, Algorithm 3 without and with compensated summation and Algorithm 3 with compensated summation using arbitrary precision software arithmetic with 32 decimal digits.

In order to explain the observed behavior, consider a one dimensional random walk with equal probability of a deviation [22], [9]. In the numerical integration process considered here, the deviation corresponds to a rounding or truncation error of one half of a unit in the last place, which is approximately $\epsilon=1.11 \times$ 


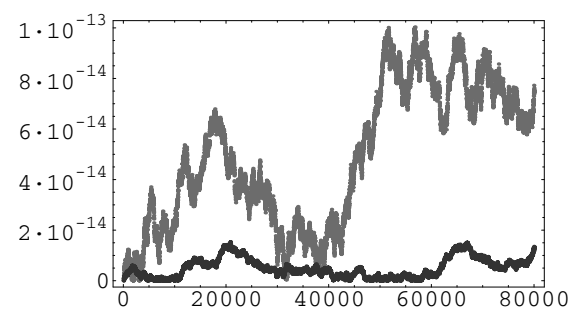

Fig. 2. Harmonic oscillator. Relative error growth vs time using Algorithm 3 without (above) and with (below) compensated summation.

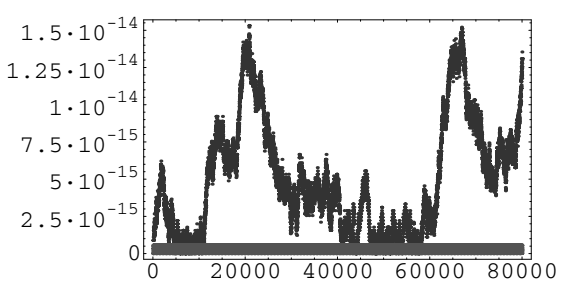

Fig. 3. Harmonic oscillator. Relative error growth vs time using Algorithm 3 with compensated summation using IEEE double precision (above) and using 32 digit software arithmetic (below).

$10^{-16}$ in IEEE double precision arithmetic. The expected absolute distance of a random walk after $N$ steps is given by $\sqrt{2 N / \pi}$.

The integration for $2 \times 10^{6}$ steps carried out with the 8th order 15 stage method in Table 1, implemented using Algorithm 1, corresponds to $N=3 \times$ $10^{7}$; therefore the expected absolute distance is $4.85 \times 10^{-13}$ which is in good agreement with the value $4.4 \times 10^{-13}$ that can be observed in Figure 1 . In the incremental Algorithm 3 the internal stages are all of the order of the step size and the only significant rounding error occurs at the end of each integration step; thus $N=2 \times 10^{6}$, leading to an expected absolute distance of $1.25 \times 10^{-13}$ which again agrees with the value $1 . \times 10^{-13}$ that can be observed in Figure 2 . This shows that for Algorithm 3, with sufficiently small step sizes, the rounding error growth is independent of the number of stages of the method, which is particularly advantageous for high order. Using compensated summation with Algorithm 3 the error growth appears to satisfy a random walk with deviation $h \epsilon$.

Similar results have been observed taking the same number of integration steps using both the 6 th order method in Table 1 , with step size $1 / 160$, and the 10th order method in [15], with the base integrator of order 2 in Table 1 and step size $1 / 10$. 


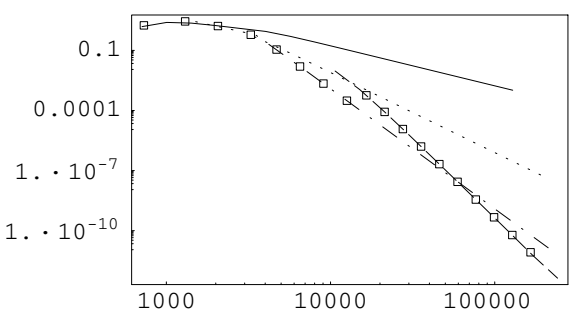

Fig. 4. Kepler problem. A log-log plot of the maximum absolute phase error vs number of evaluations of $\mathbf{f}$, using SPRK methods of order 2 (solid line), order 4 (dotted line), order 6 (dashed-dot line) and order 8 (dashed line). The methods selected automatically at various tolerances using Algorithm 4 are displayed with the symbol $\square$.

\subsection{The Kepler problem}

Kepler's problem describes the motion in the configuration plane of a material point that is attracted towards the origin with a force inversely proportional to the square of the distance. In non-dimensional form the Hamiltonian is:

$$
H(\mathbf{p}, \mathbf{q})=\frac{1}{2}\left(p_{1}^{2}+p_{2}^{2}\right)-\frac{1}{\sqrt{q_{1}^{2}+q_{2}^{2}}}
$$

The initial conditions are chosen as $p_{1}(0)=0, p_{2}(0)=\sqrt{(1+e) /(1-e)}, q_{1}(0)=$ $1-e, q_{2}(0)=0$, where the eccentricity is $e=3 / 5$. The orbit has period $2 \pi$ and the integration is carried out on the interval $[0,20 \pi]$.

Figure 4 shows some SPRK methods together with the methods chosen automatically at various tolerances according to Algorithm 4, which clearly finds a close to optimal step and order combination. The automatic selection switches to order 8 slightly earlier than necessary, which can be explained by the fact that the starting step size is based on low order derivative estimation and this may not be ideal for selecting high order methods.

Figure 5 shows the methods chosen automatically at various fixed step sizes according to Algorithm 5. With the local tolerance and step size fixed the code can only choose the order of the method. For large step sizes a high order method is selected, whereas for small step sizes a low order method is selected. In each case the method chosen minimizes the work to achieve the given tolerance.

\section{Summary and future work}

We have illustrated a few techniques that can be used to reduce the effect of cumulative rounding error in symplectic integration. If one is willing to accept the additional memory requirements, then the improved accuracy even in the low dimensional examples of Section 5 can be obtained at an increased execution time of at most a few percent. In comparison, our implementation in arbitrary 


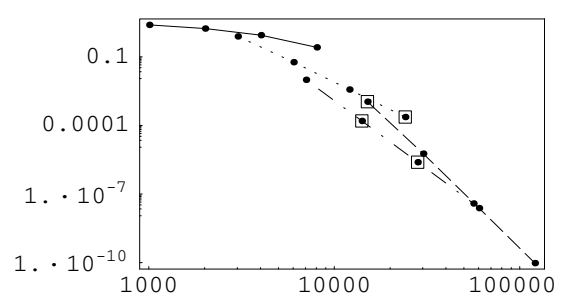

Fig. 5. Kepler problem. A log-log plot of the maximum absolute phase error vs number of evaluations of $\mathbf{f}$, using SPRK methods of order 2 (solid line), order 4 (dotted line), order 6 (dashed-dot line) and order 8 (dashed line) with step sizes $1 / 16,1 / 32,1 / 64$, 1/128. The methods selected automatically by Algorithm 5 using an absolute local error tolerance of $10^{-9}$ are displayed with the symbol $\square$.

precision using 32 decimal digit arithmetic was around an order of magnitude slower than IEEE double precision arithmetic.

Compensated summation is a general tool that can be used to improve the rounding error properties of many numerical integration methods. Furthermore, an increment formulation such as that outlined in Algorithm 3 can be beneficial if the numerical method involves a large number of basic steps. We have had similar success formulating increment algorithms for extrapolation methods. Runge-Kutta methods based on Chebyshev nodes (see [12] and [1] for a summary) also appear to be amenable to an increment formulation. We are currently investigating the application of techniques for rounding error reduction to some integration problems in celestial mechanics (see [22], [24] for example).

Algorithms for automatically selecting between a family of methods have been presented and have been shown to work well in practice. In order to test our implementation, established method coefficients have been chosen. It remains to carry out a more extensive selection amongst coefficients for better methods from recent work outlined in [2], [3], [15] [17], [18], [19].

Symplectic Runge Kutta Nyström methods are more efficient for the common class of separable Hamiltonian systems having $T(\mathbf{p})=\mathbf{p}^{T} M^{-1} \mathbf{p} / 2$, where $M$ denotes a constant symmetric matrix of masses. Moreover processing or composition techniques can be used to improve the efficiency of Runge-Kutta methods (see [4] and [2] and the references therein). Variable step size in symplectic integration has not been discussed, see [13] for a way of modifying the Hamiltonian system to accomplish this.

\section{Acknowledgements}

Thanks to Ernst Hairer for suggesting investigation of error growth using the random walk model, to Robert McLachlan for a copy of [19] and to Robert Skeel for pointing out $[6]$. 


\section{References}

1. Abdulle, A.: Chebyshev methods based on orthogonal polynomials. Ph. D. Thesis, Section de Mathématiques, Université de Genève (2001).

2. Blanes, S., Casas, F., Ros, J.: Symplectic integration with processing: a general study. SIAM J. Sci. Comput. 21 (1999) 711-727.

3. Blanes, S., Moan, P. C.: Practical symplectic partitioned Runge Kutta and Runge Kutta Nyström methods. DAMTP report NA13, Cambridge University (2000).

4. Butcher, J. C.: The numerical analysis of ordinary differential equations: Runge Kutta and general linear methods. John Wiley, Chichester (1987).

5. Candy, J., Rozmus, R.: A symplectic integration algorithm for separable Hamiltonian functions. J. Comput. Phys. 92 (1991) 230-256.

6. Earn, D. J. D., Tremaine, S.: Exact numerical studies of Hamiltonian maps: iterating without roundoff error. Physica D. 56 (1992) 1-22.

7. Forest, E., Ruth, R. D.: Fourth order symplectic integration. Physica D. 43 (1990) 105-117.

8. Gladwell, I., Shampine, L. F., Brankin, R. W.: Automatic selection of the initial step size for an ODE solver. J. Comp. Appl. Math. 18 (1987) 175-192.

9. Gladman, B., Duncan, M., Candy, J.: Symplectic integrators for long-term integrations in celestial mechanics. Celest. Mech. 52 (1991) 221-240.

10. Hairer, E., Nørsett, S. P., Wanner, G.: Solving ordinary differential equations I: nonstiff problems. 2nd edn. Springer-Verlag, New York (1993).

11. Hairer, E.: Backward analysis of numerical integrators and symplectic methods. Annals of Numerical Mathematics, 1 (1984) 107-132.

12. Hairer, E., Wanner, G.: Solving ordinary differential equations II: stiff and differential algebraic problems. 2nd edn. Springer-Verlag, New York (1996).

13. Hairer, E.: Variable time step integration with symplectic methods. Appl. Numer. Math. 25 (1997) 219-227.

14. Higham, N. J.: Accuracy and stability of numerical algorithms. SIAM, Phil. (1996).

15. Kahan, W. H., Li, R. C.: Composition constants for raising the order of unconventional schemes for ordinary differential equations. Math. Comp. 66 (1997) 10891099.

16. McLachlan, R. I., Atela, P.: The accuracy of symplectic integrators. Nonlinearity. 5 (1992) 541-562.

17. McLachlan, R. I.: On the numerical integration of ordinary differential equations by symmetric composition methods. SIAM J. Sci. Comp. 16 (1995) 151-168.

18. McLachlan, R. I.: Composition methods in the presence of small parameters. BIT. 35 (1995) 258-268.

19. McLachlan, R. I.: Families of high-order composition methods (preprint).

20. Murua, A.: On order conditions for partitioned symplectic methods. SIAM J. Numer. Anal. 34 (6) (1997) 2204-2211.

21. Okunbor, D. I., Skeel, R. D.: Explicit canonical methods for Hamiltonian systems. Math. Comp. 59 (1992) 439-455.

22. Quinn, T., Tremaine, S.: Roundoff error in long-term planetary orbit integrations. Astron. J. 99 (3) (1990) 1016-1023.

23. Sanz-Serna, J. M., Calvo, M. P.: Numerical Hamiltonian Problems. Chapman and Hall, London (1994).

24. Wisdom, J., Holman, M.: Symplectic maps for the N-body problem. Astron. J. 102 (1991) 1528-1538.

25. Yoshida, H.: Construction of high order symplectic integrators. Phys. Lett. A. 150 (1990) 262-268. 